Supplement of Foss. Rec., 24, 233-246, 2021

https://doi.org/10.5194/fr-24-233-2021-supplement

(c) Author(s) 2021. CC BY 4.0 License.

(c) (1)

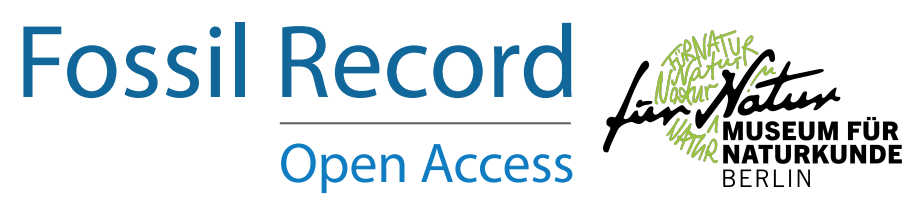

Supplement of

\title{
At the crossroads: early Miocene marine fishes of the proto-Mediterranean Sea
}

Konstantina Agiadi et al.

Correspondence to: Konstantina Agiadi (kagiadi@geol.uoa.gr)

The copyright of individual parts of the supplement might differ from the article licence. 
Otolith measurements (in mm): OL: otolith length; OH: otolith height; AL: antirostrum length; RL: rostrum length; CL: colliculum length; OCL: ostial colliculum length; CCL: caudal colliculum length; OSL: ostium length; CaL: caudal length. In addition, in Congridae only, we measured the sulcus angle $\alpha$.

\begin{tabular}{|c|c|c|c|c|c|c|c|c|c|c|c|c|c|c|c|c|}
\hline $\begin{array}{l}\text { Specimen } \\
\text { code }\end{array}$ & Species name & Sample & OL & $\mathbf{O H}$ & OL:OH & $\mathbf{A L}$ & $\mathbf{R L}$ & $\mathbf{C L}$ & CL:OL & OCL & CCL & OSL & CaL & $\begin{array}{l}\text { OSL: } \\
\text { CaL }\end{array}$ & $\alpha$ & Notes \\
\hline FEL_OT001 & $\begin{array}{l}\text { Ariosoma } \\
\text { mesohellenica }\end{array}$ & Felli 11 & 3.397 & 2.800 & 1.213 & NA & $\mathrm{NA}$ & 2.485 & 0.732 & NA & NA & NA & NA & NA & $4^{\circ}$ & $\begin{array}{l}\text { holotype AMPG } \\
2361\end{array}$ \\
\hline FEL_OT002 & $\begin{array}{l}\text { Ariosoma } \\
\text { mesohellenica }\end{array}$ & Felli 11 & 1.955 & 1.549 & 1.262 & NA & NA & 1.256 & 0.642 & NA & NA & NA & NA & NA & $2^{\circ}$ & $\begin{array}{l}\text { paratype AMPG } \\
2362\end{array}$ \\
\hline FEL_OT003 & $\begin{array}{l}\text { Ariosoma } \\
\text { mesohellenica }\end{array}$ & Felli 11 & 1.682 & 1.430 & 1.176 & NA & $\mathrm{NA}$ & 0.968 & 0.576 & NA & NA & NA & NA & NA & $2^{\circ}$ & $\begin{array}{l}\text { paratype AMPG } \\
2363\end{array}$ \\
\hline FEL_OT004 & $\begin{array}{l}\text { Ariosoma } \\
\text { mesohellenica }\end{array}$ & Felli 12 & 2.325 & 2.006 & 1.159 & NA & NA & 1.719 & 0.739 & NA & NA & NA & NA & NA & $6^{\circ}$ & $\begin{array}{l}\text { paratype AMPG } \\
2364\end{array}$ \\
\hline FEL_OT005 & Gnathophis elongatus & Felli 11 & 2.617 & 1.438 & 1.820 & NA & NA & 1.170 & 0.447 & NA & NA & NA & NA & NA & $12^{\circ}$ & $\begin{array}{l}\text { holotype AMPG } \\
2365\end{array}$ \\
\hline FEL_OT006 & Gnathophis elongatus & Felli 11 & 1.147 & 0.690 & 1.662 & NA & NA & 0.420 & 0.366 & NA & NA & NA & NA & NA & $15^{\circ}$ & $\begin{array}{l}\text { paratype AMPG } \\
2366\end{array}$ \\
\hline FEL_OT007 & Gnathophis elongatus & Felli 11 & 1.392 & 0.872 & 1.596 & NA & $\mathrm{NA}$ & 0.655 & 0.471 & NA & NA & NA & NA & NA & $10^{\circ}$ & $\begin{array}{l}\text { paratype AMPG } \\
2367\end{array}$ \\
\hline FEL_OT008 & Gnathophis elongatus & Felli 11 & 2.368 & 1.320 & 1.794 & NA & NA & 1.091 & 0.461 & NA & NA & NA & NA & NA & $20^{\circ}$ & $\begin{array}{l}\text { paratype AMPG } \\
2368\end{array}$ \\
\hline FEL_OT009 & Gnathophis elongatus & Felli 11 & 1.941 & 1.001 & 1.939 & NA & $\mathrm{NA}$ & 0.781 & 0.402 & NA & NA & NA & NA & NA & $13^{\circ}$ & $\begin{array}{l}\text { paratype AMPG } \\
2369\end{array}$ \\
\hline FEL_OT010 & Gnathophis elongatus & Felli 11 & 2.276 & 1.257 & 1.811 & NA & $\mathrm{NA}$ & 1.101 & 0.484 & NA & NA & NA & NA & NA & $15^{\circ}$ & $\begin{array}{l}\text { paratype AMPG } \\
2370\end{array}$ \\
\hline FEL_OT011 & Gnathophis elongatus & Felli 11 & 2.187 & 1.060 & 2.063 & NA & NA & 1.007 & 0.460 & NA & NA & NA & NA & NA & $11^{\circ}$ & $\begin{array}{l}\text { paratype AMPG } \\
2371\end{array}$ \\
\hline FEL_OT012 & Gnathophis elongatus & Felli 11 & 1.380 & 0.789 & 1.749 & NA & NA & 0.559 & 0.405 & NA & NA & NA & NA & NA & $20^{\circ}$ & paratype AMPG \\
\hline
\end{tabular}




\begin{tabular}{|c|c|c|c|c|c|c|c|c|c|c|c|c|c|c|c|c|}
\hline $\begin{array}{l}\text { Specimen } \\
\text { code }\end{array}$ & Species name & Sample & OL & OH & OL:OH & $\mathbf{A L}$ & $\mathbf{R L}$ & CL & CL:OL & OCL & CCL & OSL & CaL & $\begin{array}{l}\text { OSL: } \\
\text { CaL }\end{array}$ & $\alpha$ & Notes \\
\hline & & & & & & & & & & & & & & & & 2372 \\
\hline FEL_OT013 & Gnathophis elongatus & Felli 11 & NA & 1.276 & NA & NA & NA & NA & NA & NA & NA & NA & NA & NA & NA & $\begin{array}{l}\text { paratype AMPG } \\
2373\end{array}$ \\
\hline FEL_OT014 & Gnathophis elongatus & Felli 11 & 1.281 & 0.789 & 1.624 & NA & NA & 0.480 & 0.375 & NA & NA & NA & NA & $\mathrm{NA}$ & $14^{\circ}$ & $\begin{array}{l}\text { paratype AMPG } \\
2374\end{array}$ \\
\hline FEL_OT015 & $\begin{array}{l}\text { Gnathophis } \\
\text { saubriguensis }\end{array}$ & Felli 11 & 2.258 & 1.378 & 1.639 & NA & $\mathrm{NA}$ & 0.958 & 0.424 & $\mathrm{NA}$ & $\mathrm{NA}$ & NA & NA & $\mathrm{NA}$ & $18^{\circ}$ & \\
\hline FEL_OT016 & $\begin{array}{l}\text { Gnathophis } \\
\text { saubriguensis }\end{array}$ & Felli 11 & 2.083 & 1.360 & 1.532 & NA & $\mathrm{NA}$ & 1.008 & 0.484 & $\mathrm{NA}$ & $\mathrm{NA}$ & NA & NA & NA & $19^{\circ}$ & \\
\hline FEL_OT017 & $\begin{array}{l}\text { Gnathophis } \\
\text { saubriguensis }\end{array}$ & Felli 11 & 1.713 & 1.098 & 1.560 & NA & NA & 0.732 & 0.427 & $\mathrm{NA}$ & $\mathrm{NA}$ & NA & NA & NA & $14^{\circ}$ & \\
\hline FEL_OT018 & $\begin{array}{l}\text { Gnathophis } \\
\text { saubriguensis }\end{array}$ & Felli 11 & 1.461 & 0.934 & 1.564 & NA & $\mathrm{NA}$ & 0.631 & 0.432 & NA & NA & NA & NA & NA & $21^{\circ}$ & \\
\hline FEL_OT019 & $\begin{array}{l}\text { Gnathophis } \\
\text { saubriguensis }\end{array}$ & Felli 11 & 2.483 & 1.688 & 1.471 & NA & $\mathrm{NA}$ & 1.045 & 0.421 & $\mathrm{NA}$ & $\mathrm{NA}$ & $\mathrm{NA}$ & $\mathrm{NA}$ & NA & $16^{\circ}$ & \\
\hline FEL_OT020 & $\begin{array}{l}\text { Gnathophis } \\
\text { saubriguensis }\end{array}$ & Felli 11 & 3.184 & 2.336 & 1.363 & NA & $\mathrm{NA}$ & 1.645 & 0.517 & $\mathrm{NA}$ & $\mathrm{NA}$ & NA & NA & NA & $14^{\circ}$ & \\
\hline FEL_OT021 & $\begin{array}{l}\text { Gnathophis } \\
\text { saubriguensis }\end{array}$ & Felli 11 & 1.465 & 0.883 & 1.659 & NA & NA & 0.517 & 0.353 & NA & NA & NA & NA & NA & $23^{\circ}$ & \\
\hline FEL_OT022 & $\begin{array}{l}\text { Gnathophis } \\
\text { saubriguensis }\end{array}$ & Felli 11 & 2.314 & 1.502 & 1.541 & NA & NA & 0.992 & 0.429 & $\mathrm{NA}$ & $\mathrm{NA}$ & NA & NA & NA & $20^{\circ}$ & \\
\hline FEL_OT023 & $\begin{array}{l}\text { Gnathophis } \\
\text { saubriguensis }\end{array}$ & Felli 11 & 1.561 & 1.000 & 1.561 & NA & NA & 0.546 & 0.350 & NA & NA & NA & NA & NA & $21^{\circ}$ & \\
\hline FEL_OT024 & $\begin{array}{l}\text { Gnathophis } \\
\text { saubriguensis }\end{array}$ & Felli 11 & 1.828 & 1.254 & 1.458 & NA & NA & 0.681 & 0.373 & $\mathrm{NA}$ & $\mathrm{NA}$ & NA & NA & NA & $18^{\circ}$ & \\
\hline FEL_OT025 & $\begin{array}{l}\text { Gnathophis } \\
\text { saubriguensis }\end{array}$ & Felli 11 & 1.676 & 1.094 & 1.532 & NA & $\mathrm{NA}$ & 0.626 & 0.374 & $\mathrm{NA}$ & $\mathrm{NA}$ & NA & NA & NA & $24^{\circ}$ & \\
\hline
\end{tabular}




\begin{tabular}{|c|c|c|c|c|c|c|c|c|c|c|c|c|c|c|c|c|}
\hline $\begin{array}{l}\text { Specimen } \\
\text { code }\end{array}$ & Species name & Sample & OL & $\mathbf{O H}$ & OL:OH & $\mathbf{A L}$ & $\mathbf{R L}$ & $\mathbf{C L}$ & CL:OL & OCL & CCL & OSL & CaL & $\begin{array}{l}\text { OSL: } \\
\text { CaL }\end{array}$ & $\alpha$ & Notes \\
\hline FEL_OT026 & $\begin{array}{l}\text { Gnathophis } \\
\text { saubriguensis }\end{array}$ & Felli 11 & 1.776 & 1.185 & 1.499 & NA & NA & 0.755 & 0.425 & NA & NA & NA & NA & NA & $11^{\circ}$ & \\
\hline FEL_OT027 & $\begin{array}{l}\text { Gnathophis } \\
\text { saubriguensis }\end{array}$ & Felli 11 & 1.935 & 1.354 & 1.429 & NA & NA & 0.719 & 0.372 & NA & NA & NA & NA & NA & $15^{\circ}$ & \\
\hline FEL_OT028 & $\begin{array}{l}\text { Gnathophis } \\
\text { saubriguensis }\end{array}$ & Felli 11 & 1.679 & 1.089 & 1.542 & NA & NA & 0.702 & 0.418 & NA & NA & NA & NA & NA & $20^{\circ}$ & \\
\hline FEL_OT029 & $\begin{array}{l}\text { Gnathophis } \\
\text { saubriguensis }\end{array}$ & Felli 11 & 1.573 & 0.984 & 1.599 & $\mathrm{NA}$ & NA & 0.434 & 0.276 & NA & NA & NA & NA & NA & $21^{\circ}$ & \\
\hline FEL_OT030 & $\begin{array}{l}\text { Gnathophis } \\
\text { saubriguensis }\end{array}$ & Felli 12 & 2.006 & 1.323 & 1.516 & NA & NA & 0.844 & 0.421 & NA & NA & NA & NA & NA & $19^{\circ}$ & \\
\hline FEL_OT031 & $\begin{array}{l}\text { Gnathophis } \\
\text { saubriguensis }\end{array}$ & Felli 12 & 2.337 & 1.529 & 1.528 & $\mathrm{NA}$ & NA & 0.869 & 0.372 & NA & NA & NA & NA & NA & $17^{\circ}$ & \\
\hline FEL_OT032 & $\begin{array}{l}\text { Gnathophis } \\
\text { saubriguensis }\end{array}$ & Felli 12 & 3.077 & 2.127 & 1.447 & NA & NA & 1.392 & 0.452 & NA & NA & NA & NA & NA & $14^{\circ}$ & \\
\hline FEL_OT033 & Vinciguerria sp. & Felli 12 & NA & 0.649 & NA & NA & NA & NA & NA & NA & NA & NA & NA & NA & NA & \\
\hline FEL_OT034 & Vinciguerria sp. & Felli 11 & NA & 0.673 & NA & NA & NA & NA & NA & NA & NA & NA & NA & NA & NA & \\
\hline FEL_OT035 & Vinciguerria sp. & Felli 11 & NA & 1.184 & NA & NA & NA & NA & NA & NA & NA & NA & NA & NA & NA & \\
\hline FEL_OT036 & Diaphus sp. & Felli 11 & 2.535 & 1.780 & 1.424 & NA & 0.205 & NA & NA & 0.961 & 0.640 & 1.296 & 0.773 & 1.677 & NA & \\
\hline FEL_OT037 & Diaphus sp. & Felli 11 & 1.933 & 1.600 & 1.208 & 0.049 & 0.071 & NA & NA & 0.723 & 0.423 & 0.578 & 0.961 & 0.601 & NA & \\
\hline FEL_OT038 & Diaphus sp. & Felli 11 & 2.129 & 1.803 & 1.181 & 0.045 & 0.076 & NA & NA & 0.698 & 0.507 & 1.088 & 0.614 & 1.772 & NA & \\
\hline FEL_OT039 & Diaphus sp. & Felli 11 & 1.868 & 1.548 & 1.207 & NA & 0.104 & NA & NA & 0.693 & 0.322 & 1.032 & 0.438 & 2.356 & NA & \\
\hline FEL_OT040 & Diaphus sp. & Felli 11 & NA & 1.347 & NA & NA & NA & NA & NA & 0.673 & 0.468 & NA & 0.581 & NA & NA & \\
\hline FEL_OT041 & Lobianchia sp. & Felli 11 & 1.856 & 1.440 & 1.289 & 0.043 & 0.133 & NA & NA & 0.624 & 0.327 & 0.992 & 0.523 & 1.897 & NA & \\
\hline FEL_OT042 & Myripristis verus & Felli 11 & NA & NA & NA & NA & NA & NA & NA & NA & NA & NA & 4.836 & NA & NA & \\
\hline FEL_OT043 & Myripristis verus & Felli 12 & NA & 4.347 & NA & NA & NA & NA & NA & NA & 4.153 & NA & 4.494 & NA & NA & \\
\hline FEL_OT044 & Echiodon heinzelini & Felli 11 & 2.907 & 1.489 & 1.952 & NA & NA & 2.612 & 0.899 & NA & NA & NA & NA & NA & NA & \\
\hline
\end{tabular}




\begin{tabular}{|c|c|c|c|c|c|c|c|c|c|c|c|c|c|c|c|c|}
\hline $\begin{array}{l}\text { Specimen } \\
\text { code }\end{array}$ & Species name & Sample & OL & OH & OL:OH & $\mathbf{A L}$ & $\mathbf{R L}$ & CL & CL:OL & OCL & CCL & OSL & CaL & $\begin{array}{l}\text { OSL: } \\
\text { CaL }\end{array}$ & $\alpha$ & Notes \\
\hline FEL_OT045 & Apogon moyesi & Felli 11 & 2.377 & 1.794 & 1.325 & NA & NA & NA & NA & 0.874 & 0.458 & 0.988 & 0.876 & 1.128 & NA & \\
\hline FEL_OT046 & Apogon vigneauxi & Felli 11 & 3.194 & 1.988 & 1.607 & NA & NA & NA & NA & 1.172 & 0.692 & 1.355 & 1.081 & 1.253 & NA & \\
\hline FEL_OT047 & Apogon vigneauxi & Felli 11 & 3.181 & 2.387 & 1.333 & NA & NA & NA & NA & NA & NA & NA & NA & NA & NA & \\
\hline FEL_OT048 & Blennius sp. & Felli 11 & 1.002 & 0.685 & 1.463 & 0.040 & 0.148 & NA & NA & NA & NA & 0.387 & 0.249 & 1.554 & NA & \\
\hline FEL_OT049 & Arnoglossus holleri & Felli 11 & 0.948 & 0.709 & 1.337 & NA & NA & NA & NA & NA & NA & 0.457 & 0.199 & 2.296 & NA & \\
\hline FEL_OT050 & Microchirus latior & Felli 11 & 1.523 & 1.411 & 1.079 & NA & NA & NA & NA & NA & NA & 0.542 & 0.408 & 1.328 & NA & \\
\hline FEL_OT051 & Microchirus latior & Felli 11 & 1.074 & 1.113 & 0.965 & NA & NA & NA & NA & NA & $\mathrm{NA}$ & 0.440 & 0.241 & 1.826 & NA & \\
\hline FEL_OT052 & Microchirus latior & Felli 12 & 1.686 & 1.645 & 1.025 & NA & NA & NA & NA & NA & NA & 0.521 & 0.669 & 0.779 & NA & \\
\hline FEL_OT053 & Mullus elongatus & Felli 11 & 2.558 & 1.451 & 1.763 & NA & 0.417 & NA & NA & NA & NA & 0.461 & 1.376 & 0.335 & NA & \\
\hline FEL_OT054 & Mullus elongatus & Felli 11 & 4.188 & 2.302 & 1.819 & NA & NA & NA & NA & NA & NA & 0.804 & 2.620 & 0.307 & NA & \\
\hline FEL_OT055 & Mullus elongatus & Felli 12 & NA & 2.208 & NA & NA & NA & NA & NA & NA & NA & NA & NA & NA & NA & \\
\hline FEL_OT056 & Mullus elongatus & Felli 12 & 1.214 & 0.689 & 1.762 & NA & NA & NA & NA & NA & NA & NA & NA & NA & NA & \\
\hline FEL_OT057 & Cepola yrieuensis & Felli 11 & 4.410 & 2.566 & 1.719 & NA & 0.667 & NA & NA & NA & NA & 1.661 & 1.051 & 1.580 & NA & \\
\hline FEL_OT058 & Cepola yrieuensis & Felli 11 & 3.697 & 2.064 & 1.791 & NA & 0.567 & NA & NA & NA & NA & 1.510 & 0.875 & 1.726 & NA & \\
\hline FEL_OT059 & Pomadasys sp. & Felli 11 & 2.372 & 1.545 & 1.535 & NA & 0.354 & NA & NA & 0.709 & NA & 0.973 & 0.902 & 1.079 & NA & \\
\hline FEL_OT060 & Pomadasys sp. & Felli 11 & 2.027 & 1.310 & 1.547 & NA & 0.364 & NA & NA & 0.540 & NA & 0.750 & 0.941 & 0.797 & NA & \\
\hline FEL_OT061 & Pomadasys sp. & Felli 11 & 1.429 & 0.970 & 1.473 & NA & 0.190 & NA & $\mathrm{NA}$ & NA & NA & NA & NA & NA & NA & \\
\hline FEL_OT062 & Pomadasys sp. & Felli 11 & 2.190 & 1.481 & 1.479 & NA & 0.479 & NA & NA & 0.605 & NA & 0.863 & 0.969 & 0.891 & NA & \\
\hline FEL_OT063 & Pomadasys sp. & Felli 11 & 1.894 & 1.209 & 1.567 & NA & 0.297 & NA & NA & 0.406 & NA & 0.520 & 0.933 & 0.557 & NA & \\
\hline FEL_OT064 & Pomadasys sp. & Felli 11 & 1.875 & 1.270 & 1.476 & NA & 0.426 & NA & NA & 0.330 & NA & 0.559 & 0.899 & 0.622 & NA & \\
\hline FEL_OT065 & Pagellus sp. & Felli 11 & 3.475 & 2.101 & 1.654 & NA & 0.487 & NA & NA & 0.877 & NA & 1.232 & 1.372 & 0.898 & NA & \\
\hline FEL_OT066 & Pagellus sp. & Felli 12 & 2.223 & 1.502 & 1.480 & NA & 0.352 & NA & NA & NA & NA & 0.725 & 1.158 & 0.626 & NA & \\
\hline FEL_OT067 & Pagrus sp. & Felli 11 & NA & 1.782 & NA & NA & NA & NA & NA & NA & NA & NA & 1.326 & NA & NA & \\
\hline FEL_OT068 & Spicara cf. gossei & Felli 11 & 2.618 & 1.539 & 1.701 & 0.083 & 0.409 & NA & $\mathrm{NA}$ & NA & NA & 0.816 & 0.931 & 0.876 & NA & \\
\hline
\end{tabular}




\begin{tabular}{|c|c|c|c|c|c|c|c|c|c|c|c|c|c|c|c|c|}
\hline $\begin{array}{l}\text { Specimen } \\
\text { code }\end{array}$ & Species name & Sample & OL & OH & \begin{tabular}{|l|} 
OL:OH \\
\end{tabular} & $\mathbf{A L}$ & RL & CL & CL:OL & OCL & CCL & OSL & $\mathrm{CaL}$ & $\begin{array}{l}\text { OSL: } \\
\text { CaL }\end{array}$ & $\alpha$ & Notes \\
\hline FEL_OT069 & Spicara cf. gossei & Felli 11 & 0.827 & 0.607 & \begin{tabular}{|l|}
1.362 \\
\end{tabular} & NA & 0.269 & NA & NA & $\mathrm{NA}$ & NA & NA & NA & NA & NA & \\
\hline FEL_OT070 & Spicara cf. gossei & Felli 11 & 0.966 & 0.665 & 1.453 & NA & 0.204 & NA & NA & NA & NA & 0.237 & 0.378 & 0.627 & NA & \\
\hline FEL_OT071 & Spicara cf. gossei & Felli 11 & NA & 1.781 & NA & NA & NA & NA & NA & NA & NA & 0.835 & 1.047 & \begin{tabular}{|l|}
0.798 \\
\end{tabular} & NA & \\
\hline FEL_OT072 & Spicara cf. gossei & Felli 11 & 1.650 & 0.959 & 1.721 & NA & 0.307 & NA & NA & NA & NA & 0.369 & 0.781 & 0.472 & NA & \\
\hline FEL_OT073 & Spicara cf. gossei & Felli 11 & 1.262 & 0.849 & 1.486 & $\mathrm{NA}$ & 0.306 & $\mathrm{NA}$ & $\mathrm{NA}$ & $\mathrm{NA}$ & $\mathrm{NA}$ & 0.369 & 0.547 & \begin{tabular}{|l}
0.675 \\
\end{tabular} & NA & \\
\hline FEL_OT074 & Spicara cf. gossei & Felli 11 & 1.628 & 1.148 & 1.418 & NA & 0.382 & NA & NA & NA & NA & 0.431 & 0.678 & 0.636 & NA & \\
\hline FEL_OT075 & Spicara cf. gossei & Felli 11 & 1.839 & 1.131 & \begin{tabular}{|l|}
1.626 \\
\end{tabular} & NA & 0.491 & $\mathrm{NA}$ & NA & NA & NA & 0.555 & 0.692 & 0.802 & NA & \\
\hline FEL_OT076 & Spicara cf. gossei & Felli 11 & NA & 0.809 & NA & $\mathrm{NA}$ & NA & $\mathrm{NA}$ & $\mathrm{NA}$ & $\mathrm{NA}$ & $\mathrm{NA}$ & NA & 0.400 & NA & NA & \\
\hline FEL_OT077 & Spicara cf. gossei & Felli 12 & 1.639 & 1.167 & 1.404 & NA & 0.414 & NA & NA & NA & NA & 0.388 & 0.696 & 0.557 & NA & \\
\hline FEL_OT078 & Spicara cf. gossei & Felli 12 & 1.561 & 0.995 & 1.569 & NA & 0.431 & NA & NA & NA & NA & 0.507 & NA & NA & NA & \\
\hline FEL_OT079 & Spicara cf. gossei & Felli 12 & 1.643 & 0.994 & 1.653 & NA & 0.376 & NA & NA & NA & NA & 0.510 & 0.679 & 0.751 & NA & \\
\hline FEL_OT080 & Spicara cf. gossei & Felli 12 & NA & 0.979 & NA & $\mathrm{NA}$ & NA & NA & NA & NA & $\mathrm{NA}$ & 0.368 & 0.717 & \begin{tabular}{|l|l|}
0.513 \\
\end{tabular} & NA & \\
\hline FEL_OT081 & Spicara cf. gossei & Felli 12 & 0.920 & 0.633 & \begin{tabular}{|l|}
1.453 \\
\end{tabular} & NA & 0.228 & $\mathrm{NA}$ & NA & NA & NA & 0.336 & 0.313 & \begin{tabular}{|l|}
1.073 \\
\end{tabular} & NA & \\
\hline FEL_OT082 & Spicara cf. gossei & Felli 12 & 1.262 & 0.882 & 1.431 & NA & 0.277 & NA & NA & NA & NA & 0.301 & 0.545 & 0.552 & NA & \\
\hline FEL_OT083 & Spicara cf. gossei & Felli 12 & NA & 1.231 & NA & NA & NA & NA & NA & NA & NA & 0.548 & 0.974 & \begin{tabular}{|l|l|}
0.563 \\
\end{tabular} & NA & \\
\hline
\end{tabular}

